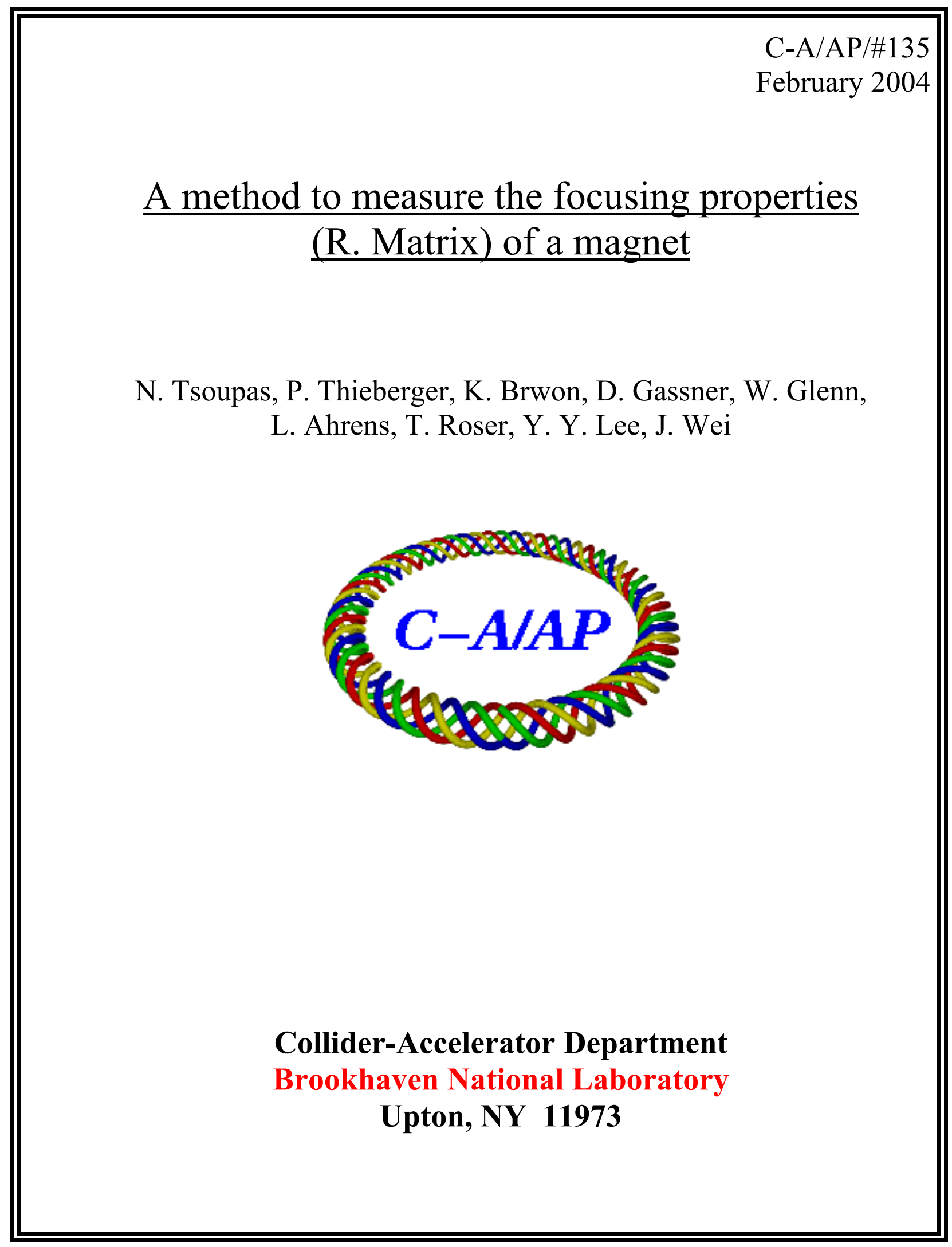




\title{
A Method to Measure the focusing properties (R_Matrix) of a magnet
}

\author{
N. Tsoupas, P. Thieberger, K. Brown, D. Gassner, W. Glenn, L. Ahrens, T. Roser, \\ Y.Y. Lee, J. Wei
}

\begin{abstract}
In this technical note we discuss, and study the feasibility of a method that may be used to measure the focusing properties of a magnet. This method may prove valuable when applied to "non_conventional" magnets that deviate from the usual dipole magnets or other multipole magnets (quadrupoles /sextupoles etc.) which are commonly used in a synchrotron. In this category of "non_conventional" magnets, fall special magnets, which come under the name "Snakes". Such magnets are being used in synchrotron accelerators[1,2] to introduce artificial spin resonances to help overcome the intrinsic and/or imperfection spin resonances which appear during the acceleration of polarized beams. Such spin resonances are the cause of some polarization loss that occurs during the acceleration of the polarized beam in AGS.

This method of measuring the focusing properties of a magnet requires the use of "low energy" and "high rigidity" heavy-ions which may be obtained from the BNL Tandem accelerator.

In brief the method consists on, injecting "narrow_beamlets" of heavy ions into a magnet and measuring the coordinates, of these "narrow_beamlets", at the entrance and exit of the magnet.

From the measurement of these coordinates of the "narrow_beamlets" we can deduce information on the R matrix (first order transfer matrix elements) and higher order matrix elements that define the focusing properties of the magnet.
\end{abstract}

\section{Introduction}

The common method, which is applied in the magnet division of the Brookhaven National Laboratory (BNL), to determine the focusing properties of a magnet, is to measure the magnetic field of the magnet by using various techniques. Few of the techniques which are utilized to measure the magnetic field of a magnet are listed below:

a) Using Hall probes to map the field components $\left(B_{x}, B_{y}, B_{z}\right)$ of a magnet on each of the points of a three dimensional rectangular grid which is fixed relative to the magnet. This three dimensional magnetic field map can then be used in conjunction with other computer codes to determine the focusing properties of the magnet.

b) Using a "Long rotating" coil that measures the integrated multipoles of the radial component of the magnetic field. The integrated multipoles can then be used as input values for the strengths $\left(L \cdot K_{1}\right.$ for quadrupole, $L \cdot K_{2}$ for sextupole, $L \cdot K_{3}$ for octupole etc.) of the various multipoles.

c) Using a "Short rotating coil" which measures the magnetic multipoles of the radial component of the magnetic field along the length of the magnet.

d) Using a long "Flipping coil" that measures one of the integrated multipole component of the magnetic field. This technique is usually applied to measure the integrated strength $\int \mathrm{B}_{\mathrm{y}} \mathrm{ds}$ of a dipole magnet to determine its Integral Transfer Function (ITF). 
The magnetic field measurements techniques (a to $\mathrm{d}$ above) provide accurate results (with a relative error $\leq 10^{-4}$ ) when the method is applied to conventional magnets, like dipoles or higher order multipoles.

The magnetic-field measurement method however is not very accurate to measure the focusing properties of a "non_conventional" magnet, like a Siberian Snake[3,4].

In this technical note we study the possibility of using "low energy" but "high rigidity" heavy ions to measure the focusing properties of a Helical Snake[3,4] or any other magnet that does not lend itself to any of the magnetic measurements technique mentioned earlier. The following issues (see (a) to (e) below), regarding the proposed method, will be discussed below.

a) Principle of the method to measure the focusing properties of a magnet.

b) Description of the device which can be used to measures the focusing properties of a magnet.

c) Production of heavy ions to be used with the device to measure the focusing properties of a magnet.

d) Details on how to apply the method to measure the focusing properties of a magnet.

e) Calculation of the physical quantities (R_matrix) that characterize the optical properties of the magnet and estimation of the errors on these physical quantities due to the measurement error.

\section{Principle of the Method to measure the focusing properties of a magnet}

The focusing properties of a magnet are considered known when the coordinates of a particle at the exit of a magnet can be determined, assuming that the coordinates of the particle at the entrance of the magnet are known. The above sentence can be expressed either, schematically in Figure 1 which shows a magnet with the coordinates of a particle at the entrance and exit coordinate systems or mathematically in equation (1) below.
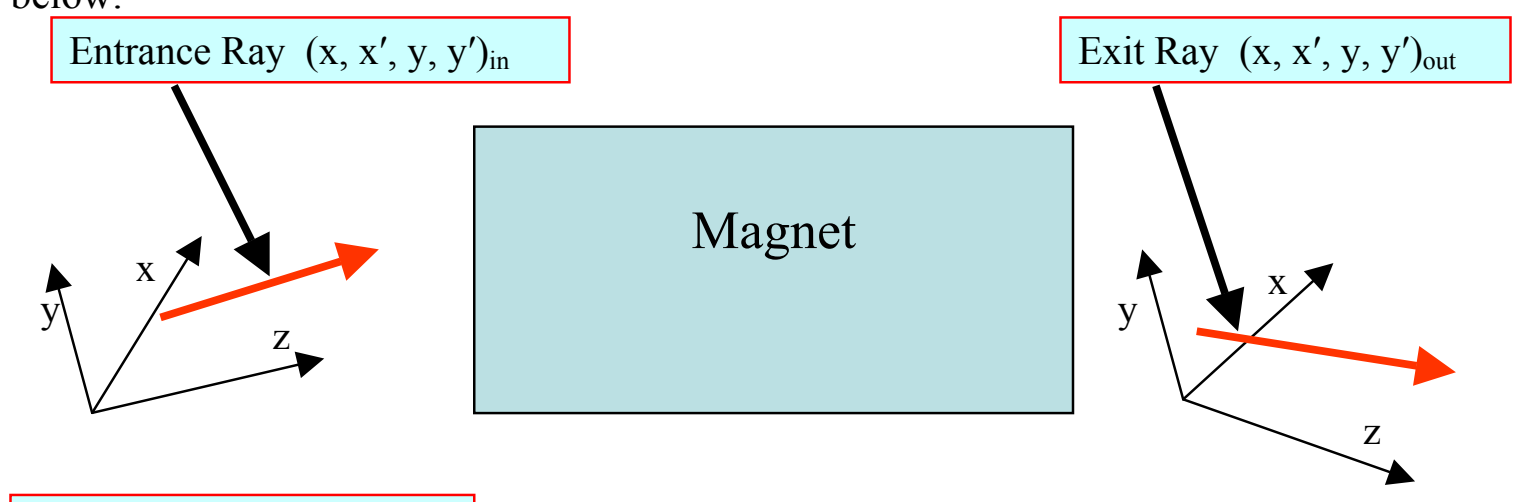

Entrance Coord. System

Exit Coord. System

Figure 1. Schematic diagram of a magnet with the entrance end exit coordinate system. The starting point of the red arrows corresponds to the position of the particle, and the direction of the arrow shows the direction of the particle at the entrance/exit coordinate systems. respectively. 


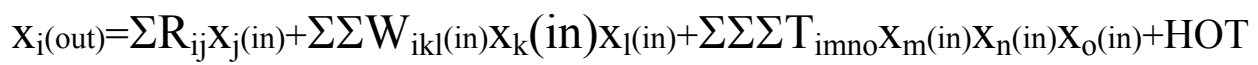

The notation in equation (1) is: $\left(\mathrm{x}_{1}, \mathrm{x}_{2}, \mathrm{x}_{3}, \mathrm{x}_{4}, \mathrm{x}_{5}, \mathrm{x}_{6}\right)<=>\left(\mathrm{x}, \mathrm{x}^{\prime}, \mathrm{y}, \mathrm{y}^{\prime}, \mathrm{d} l, \delta \mathrm{p} / \mathrm{p}\right)$

Where $x, y, x^{\prime}, y^{\prime}$ are the lateral $(x, y)$ and angular $\left(x^{\prime}, y^{\prime}\right)$ deviations of the particle from the trajectory of the central particle. The quantity $\delta p$ is the momentum deviation of the particle's momentum $\mathrm{p}$ from the momentum $\mathrm{p}_{0}$ of the central-particle, and $\delta l$ is the path length difference of the particle's path from the path of the central particle.

The coefficients in the expansion (1) are defined as the partial derivatives of the output coordinates with respect to the input coordinates (shown below).

$\begin{array}{ll}\mathrm{R}_{\mathrm{ij}}=\partial \mathrm{x}_{\mathrm{i}} / \partial \mathrm{x}_{\mathrm{j}}, & 1^{\text {st }} \text { order } \\ \mathrm{W}_{\mathrm{ikl}}=\partial \mathrm{x}_{\mathrm{i}} /\left(\partial \mathrm{x}_{\mathrm{k}} \partial \mathrm{x}_{\mathrm{l}}\right) & 2^{\text {nd }} \text { order } \\ \mathrm{T}_{\mathrm{imno}}=\partial \mathrm{x}_{\mathrm{i}} /\left(\partial \mathrm{x}_{\mathrm{m}} \partial \mathrm{x}_{\mathrm{n}} \partial \mathrm{x}_{\mathrm{o}}\right) & 3^{\text {rd }} \text { order } \\ \ldots \ldots \ldots \ldots \ldots \ldots & \ldots \ldots \ldots \ldots \\ \mathrm{T}_{\text {imno... }}=\partial \mathrm{x}_{\mathrm{i}} /\left(\partial \mathrm{x}_{\mathrm{m}} \partial \mathrm{x}_{\mathrm{n}} \partial \mathrm{x}_{\mathrm{o}} \ldots \partial \mathrm{x}_{\mathrm{p}}\right) & \mathrm{n}^{\text {th }} \text { order }\end{array}$

The knowledge of the coefficients $\mathrm{R}_{\mathrm{ij}}, \mathrm{W}_{\mathrm{ijl}}, \mathrm{T}_{\mathrm{imno}}, \ldots .$. and of the higher order coefficients, appearing in the Higher Order Terms (HOT) determine the focusing properties of any magnet.

In the "paraxial ray approximation", which assumes that the momentum deviation $\delta p$ of the momentum $\mathrm{p}$ of the particle is much smaller than momentum $\mathrm{p}_{0}\left(\delta \mathrm{p} / \mathrm{p}_{0} \leq 0.01\right)$ of the central particle, and that the lateral coordinates $\mathrm{x}, \mathrm{y}$ in both entrance and exit coordinate systems are much smaller than the radius of curvature ${ }^{1} \rho$, of the orbit of a particle moving in the magnetic field $B$ of the magnet, most of the higher than the $1^{\text {st }}$ order terms which appear in equation (1) $\left(\mathrm{W}_{\mathrm{ikl}}, \mathrm{T}_{\mathrm{imno}}\right.$....etc) are usually negligible. Therefore, the equation (1) above can be written as:

$$
\mathrm{X}_{\mathrm{i} \text { (out) }}=\Sigma \mathrm{R}_{\mathrm{ij}} \mathrm{X}_{\mathrm{j}} \text { (in) }+ \text { "few HOT". }
$$

In equation (2) the expression "few HOT" will be clarified in a subsequent section. In summary, the first order coefficients $\mathrm{R}_{\mathrm{ij}}$, and the "most important" higher order coefficients appearing in equations $(1,2)$ can be computed by measuring the coordinates $\left(\mathrm{x}, \mathrm{x}^{\prime}, \mathrm{y}, \mathrm{y}^{\prime}\right)_{\text {exit }}$ of an "adequate" number of particles at the exit of the magnet and the corresponding coordinates $\left(\mathrm{x}, \mathrm{x}^{\prime}, \mathrm{y}, \mathrm{y}^{\prime}\right)_{\text {in }}$ of the particles at the entrance coordinate system. By solving equation (2) we can determine the first order $\mathrm{R}_{\mathrm{ij}}$, and the "most important" higher order coefficients.

\section{Description of the proposed apparatus that can be used to determine the focusing properties of a magnet}

A schematic diagram of the proposed apparatus which can be used to measure the focusing properties of a magnet is shown in Figure 2.

\footnotetext{
${ }^{1}$ The radius of curvature $\rho$ is defined in the equation $B \cdot \rho=k \cdot p / Q$ (B is the field of the magnet, $p$ is the momentum, and $\mathrm{Q}$ is the charge state of the ion, $\mathrm{k}$ is a constant which depends on the units),
} 


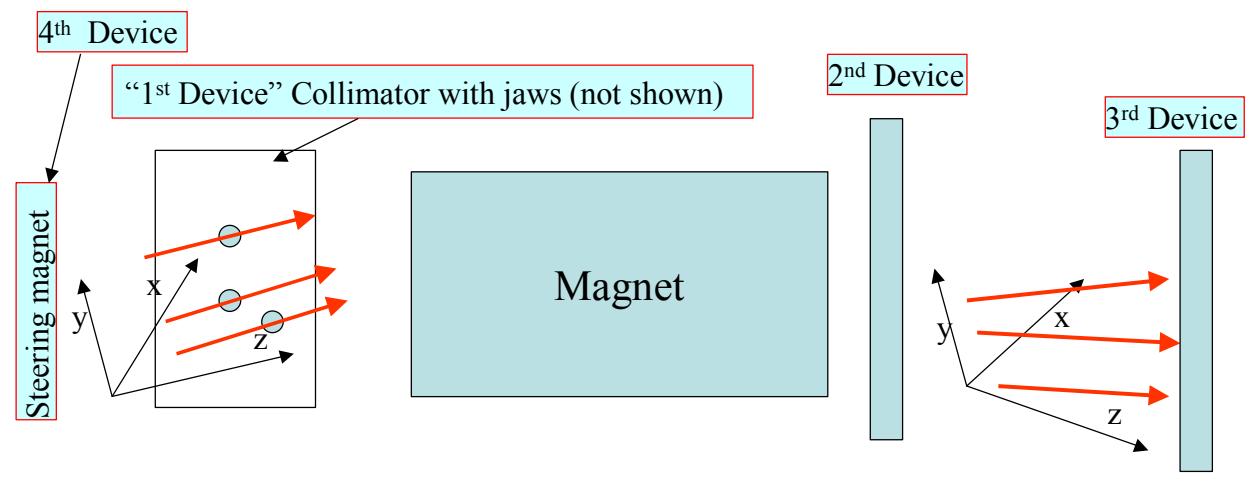

Figure 2. Schematic diagram of the magnet with the various devices which define the position $(\mathrm{x}, \mathrm{y})$ and direction $\left(\mathrm{x}^{\prime}, \mathrm{y}^{\prime}\right)$ of the rays at the entrance and exit of the magnet.

The apparatus can be considered to consist of four separate devices:

a) The first device is the " 1 st ion-position defining device" which is placed at the entrance of the magnet and is located at a specified distance from the magnet iron, just before the start of the fringe field of the magnet.

The " 1 st ion-position defining device" consist of a metal plate, labeled as "collimator" shown in Figure 2, which is thick enough to stop the incident ions.

The collimator plate has "pin holes", through which the ions will pass to enter the magnet. The location of the center of the pin holes will define the location $(x, y)_{\text {in }}$ of the particles at the entrance.

Two sets of "jaws", one horizontal and one vertical which can move parallel to the surface of the "collimator" will allow to select one pin hole at a time that the ions will enter the magnet. If the "jaw system" has an additional degree of freedom which allows the "jaw system" to rotate about the longitudinal axis (z-axis), this will permit the selection of a set of pin-holes which are lined up along any direction on the collimator plate.

The "jaws" should be thick enough to stop the particles entering the magnet.

b) The second device is the " $2^{\text {nd }}$ ion-position measuring device" which is located at a specified distance from the magnet iron at the exit of the magnet, just after the fringe field of the magnet. The " 2 nd ion-position measuring device" consists of two thin wires, at right angles with each other.

Each wire can move with respect to the other wire and maintain their perpendicularity.

The position of each wire should be known with accuracy of $\sim 0.050 \mathrm{~mm}$.

It is desirable that both wires rotate about the z-axis while maintaining the perpendicularity in the wires. This rotation will allow the detection of the individual rays

\footnotetext{
${ }^{2}$ The size of the holes depends on the magnet to be measured. For the Partial Snake magnet the diameter of the holes should be no greater than $0.5 \mathrm{~mm}$.
} 
at the exit of the magnet while many rays (emerging from different pin-holes at the entrance collimator) are entering the magnet simultaneously.

Both wires will help determine the position of the centroid of a set of particles which emerge from a particular pin-hole. Each centroid corresponds to the position coordinates $(\mathrm{x}, \mathrm{y})_{\text {out }}$ of the rays at the exit.

The direction of the rays $\left(\mathrm{x}^{\prime}, \mathrm{y}^{\prime}\right)_{\text {in }}$ at the entrance of the magnet can be determined from the coordinates $(\mathrm{x}, \mathrm{y})_{\text {in }}$ of the rays at the entrance, as defined by the " 1 st ion-position defining device", and the coordinates $(\mathrm{x}, \mathrm{y})_{\text {out }}$ of the rays at the exit as measured by the " 2 nd ion-position measuring device" when the field of the magnet is off.

c) The third device is named as the " 3 rd ion-position measuring device" and is located at the exit of the magnet at a specified distance, along the z-axis, from the location of " 2 nd ion-position measuring device" otherwise it is identical to the " 2 nd ionposition measuring device". Position measurements of the ions taken from the $2^{\text {nd }}$ and $3^{\text {rd }}$ " ion-position measuring devices" will determine the direction of the ions at the exit of the magnet $\left(\mathrm{x}^{\prime}, \mathrm{y}^{\prime}\right)_{\text {out }}$.

d) The fourth device is the "ray-direction defining" magnet which is a dipole magnet that can change the direction $\left(\mathrm{x}^{\prime}, \mathrm{y}^{\prime}\right)$ in of the rays at the entrance of the magnet and also serves to select the charge-state to be used when using heavy ions.

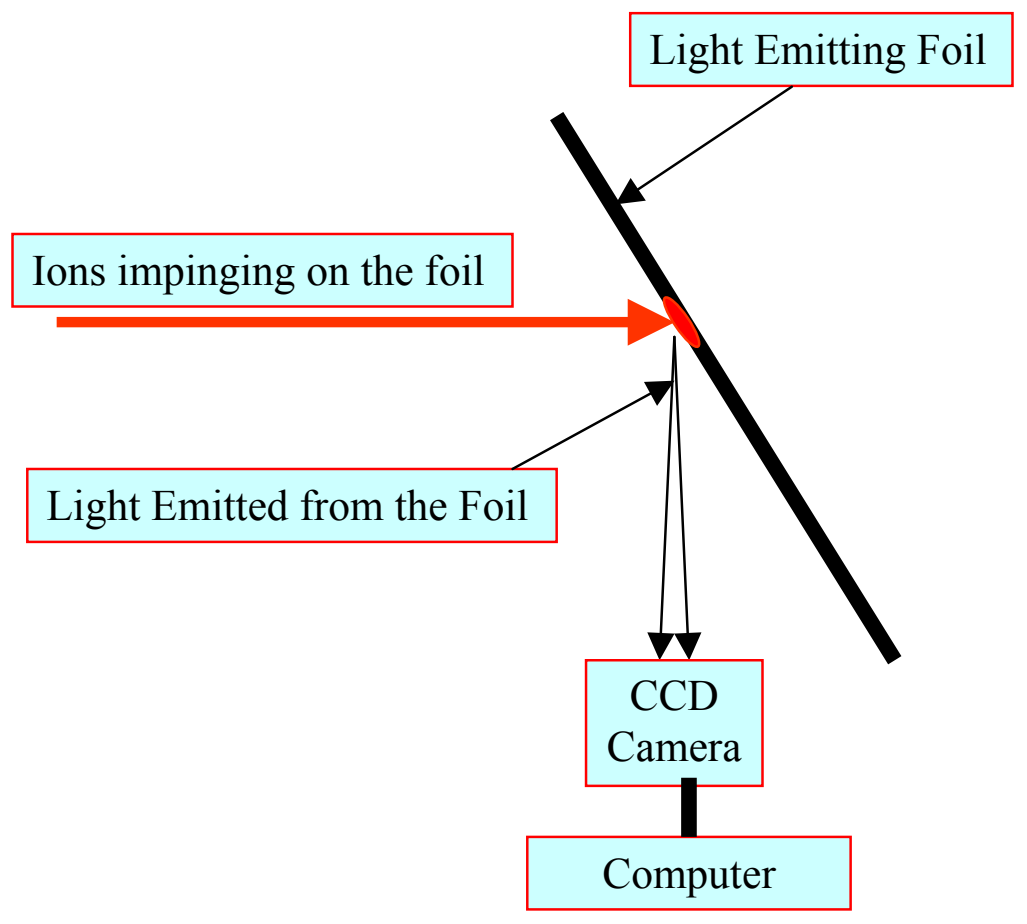

Figure 3. Schematic diagram of an ion-position measuring system to be used in the position measurement of the pencil-like ion beams which will enter and exit the magnet. 
An alterative system which can be used instead of the $2^{\text {nd }}$ and $3^{\text {rd }}$ position measuring devices described above, consists of a light emitting foil (visual Flag) and a CCD camera connected to a computer. The light emitting foil is a compound of Gadolinium Oxy-Sulfide doped with Terbium (Gd2O2S:Tb). The compound is bonded on an Al foil.

The ions emerging from the pin holes of the " 1 st ion-position defining device" will generate light upon their impact on the foils of the $2^{\text {nd }}$ or $3^{\text {rd }}$ measuring devices.

The emitted light will be detected by the CCD camera and the computer will calculate the location of each "pin-hole image" on the visual flags (foils).

A schematic diagram of the detection system is shown in Fig. 3. This ion-position detection system seems to be superior to the wire system described earlier.

\section{How to obtain the "slow speed high rigidity" heavy ions}

In order to determine the optical properties of a magnet, at the excitation field that the magnet will be used in the AGS, we should inject into the magnet heavy ions that have comparable rigidity to the rigidity of the polarized protons in AGS at injection energies.

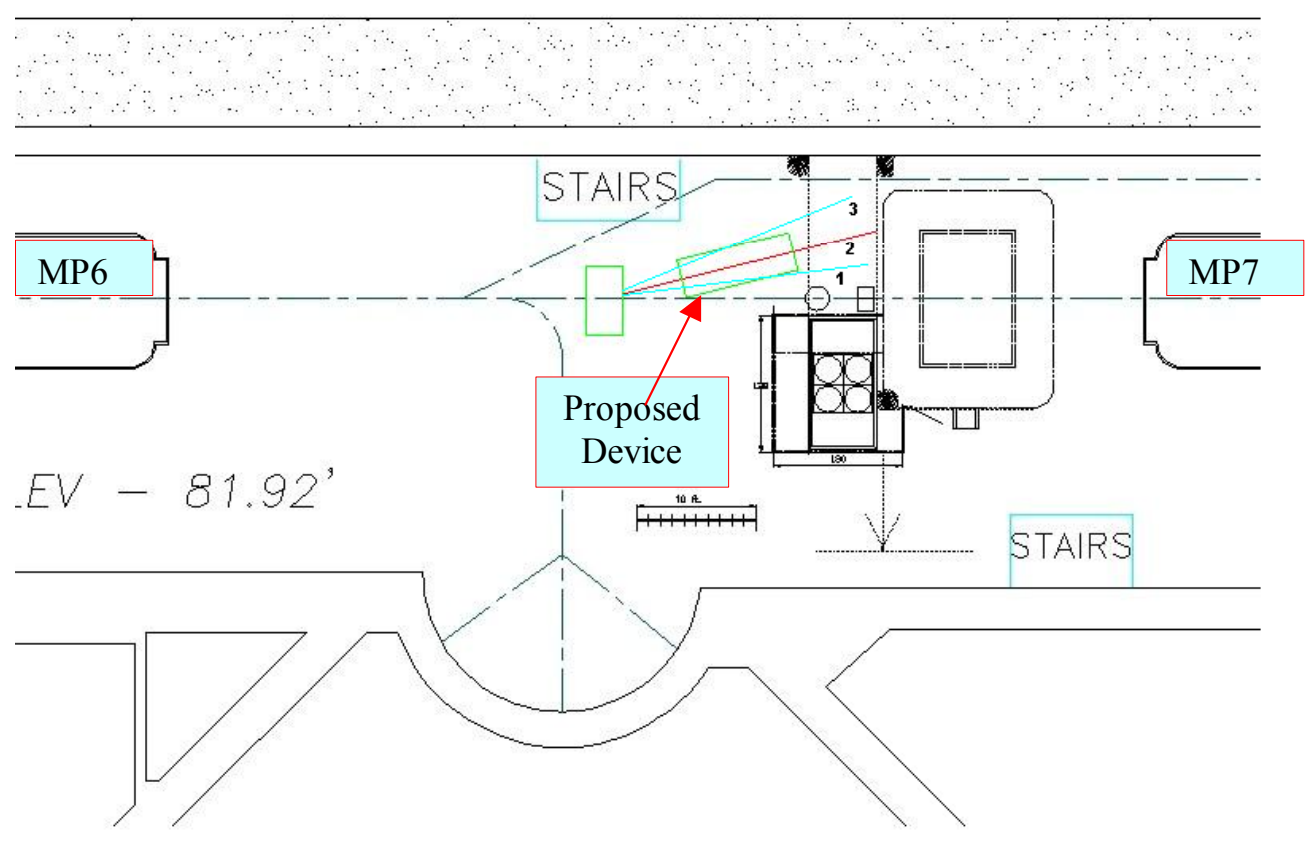

Figure 4. Possible location of the proposed magnet measuring system located between the MP6 and MP7 Tandem accelerators. Heavy ions from MP6 are deflected by a dipole to separate the charge states indicated by the numbers 1,2 and 3. For example gold ions of charge states 1 or 2 could be used (see text) 
In order to eliminate radiation problems and nuclear activation of the detection system, it is advisable to use heavy ions with both, very high Mass (A) and Atomic Number $(Z)$ which are at the lowest possible ionization state $(+1$ or +2$)$.

Table 1 contains the physical quantities associated with the polarized protons (third row) which are injected into the AGS synchrotron, and few candidates of heavy ions that have the same rigidity as the polarized protons (eighth column) and can be produced in the BNL Tandem.

The last row of Table 1 is referred to $\mathrm{Cs}+$ ions that can be produced with a $60 \mathrm{keV}$ ion source to be described elsewhere[7].

Figure 4 is a drawing of the location inside the Tandem building where the apparatus can be placed.

TABLE 1. Physical quantities associated with various ions ( rows $4^{\text {th }}, 5^{\text {th }}, 6^{\text {th }}$ ) which have comparable rigidities to the rigidities of the polarized protons $\left(3^{\text {rd }}\right.$ row $)$ when injected in AGS. The last row corresponds to Cs+ ions that can be produced with a $60 \mathrm{kV}$ ion source[7] but with much lower rigidity than that required to determine the optical properties of the Partial Snake at the operating magnetic field of the Snake.

\begin{tabular}{|c|c|c|c|c|c|c|c|c|}
\hline Species & A & $\mathrm{Z}$ & $\begin{array}{l}\text { Charge } \\
\text { State }\end{array}$ & $\gamma$ & $\begin{array}{c}\mathrm{T} \\
{[\mathrm{GeV}]}\end{array}$ & $\begin{array}{c}\mathrm{p} \\
{[\mathrm{GeV} / \mathrm{c}]}\end{array}$ & $\begin{array}{c}\mathrm{B} \rho \\
{[\mathrm{T} \cdot \mathrm{m}]}\end{array}$ & Production \\
\hline $\mathrm{p}$ & 1 & 1 & +1 & 2.62 & 1.520 & 2.272 & 7.590 & BNL-LINAC \\
\hline $\mathrm{Au}$ & 197 & 79 & +1 & 1.0000757 & 0.014 & 2.272 & 7.590 & TANDEM \\
\hline $\mathrm{Au}$ & 197 & 79 & +2 & 1.0002273 & 0.042 & 3.935 & 6.573 & TANDEM \\
\hline $\mathrm{U}$ & 238 & 92 & +1 & 1.0000519 & 0.014 & 2.272 & 7.590 & TANDEM \\
\hline Cs & 133 & 55 & +1 & 1.0000006 & 0.000 & 0.141 & 0.471 & Ion_Source \\
\hline
\end{tabular}

\section{How to apply the method to measure the focusing properties of a magnet}

The method to determine the focusing properties of a magnet is based in the determination of the coefficients appearing in equation (1). The total number of the first second and third order coefficients (matrix elements $\mathrm{R}_{\mathrm{ij}}, \mathrm{T}_{\mathrm{ijk}}, \mathrm{W}_{\mathrm{ijkl}}$ ) appearing in equation (1) is 588. Some of the 588 matrix elements are identical to zero[5]. By injecting 102 input rays into the magnet, appropriately chosen[5], one can generate enough equations like equation (1) to determine all the matrix elements up to third order. For a magnet that the "paraxial approximation" is valid, only few of the higher order matrix elements are significant.

By injecting 22 input rays[5] into the magnet, all the first order matrix elements, and few or the second order matrix elements can be calculated.

It is very important however to determine theoretically, before the experimental determination of the focusing properties of the magnet, which of the matrix elements are significant (their contribution is of the same order of magnitude as the first order matrix elements). This determination is being done using theoretical ray-tracing through the magnet whose magnetic field has been calculated in three dimensions by the use of 3D modeling. In this example we use the computer code of Vector Fields[8] to calculate the 
three dimensional magnetic field $\left(\mathrm{B}_{\mathrm{x}}, \mathrm{B}_{\mathrm{y}}, \mathrm{B}_{\mathrm{z}}\right)$ of the Partial Snake. Reference [4] provides information on the most important matrix elements of a helical dipole partial snake whose focusing properties we propose to measure.

The procedure we followed to determine the significant Transfer-matrix elements of a magnet is outlined below.

a) We model the magnet in 3 dimensions. A computer code calculates the three components of the magnetic fields $\left(B_{x}, B_{y}, B_{z}\right)$, which is based on the $3 D$ model of the magnet.

b) The theoretically computed magnetic fields in step (a) above are utilized as an input in a computer code[6] which "ray-traces" ions with specific initial conditions (known coordinates of the ion at the entrance of the magnet), to calculate the coordinates of these ions at the exit of the magnet. Given an adequate number of input rays we can generate enough equations similar to equation $(1,2)$ above to calculate the Transfer-matrix elements using the computer code[6].

c) Determine the error in the matrix elements due to the measurement error in the position and angle of the particles.

d) Determine which of the matrix elements are important by comparing the theoretically calculated matrix elements in step (b) with the error of the matrix elements due to the measurements.

e) Keep the important matrix elements in equation (2) and proceed with the experimental measurements.

f) Select a convenient set of ray-coordinates $\left(x, x^{\prime}, y, y^{\prime}\right)$ in at the entrance of the magnet. This set of rays can be similar to the one used in the theoretical calculation in step (b) above. Measure the coordinates $\left(\mathrm{x}, \mathrm{x}^{\prime}, \mathrm{y}, \mathrm{y}^{\prime}\right)_{\text {in }}$ of the ionrays at the entrance and the coordinates of the ions $\left(\mathrm{x}, \mathrm{x}^{\prime}, \mathrm{y}, \mathrm{y}^{\prime}\right)$ exit at the exit of the magnet.

g) Use the measured coordinates $\left(\mathrm{x}, \mathrm{x}^{\prime}, \mathrm{y}, \mathrm{y}^{\prime}\right)_{\text {in }}$ and $\left(\mathrm{x}, \mathrm{x}^{\prime}, \mathrm{y}, \mathrm{y}^{\prime}\right)_{\text {exit }}$ of the various rays as input in equation (2) to solve for the first order Transfer-matrix elements and the most significant higher order Transfer-matrix elements.

\section{The most important Matrix elements for a Partial Snake and the corresponding error in the matrix elements due to the measurement error.}

The calculations performed with the computer code SPRAY[6] yielded the following results for the first order matrix elements ("Ideal R_matrix").

\begin{tabular}{rrrrrr}
\multicolumn{7}{c}{ *TRANSFORM } & Units in m,rads \\
0.92028 & 3.11399 & -0.00885 & -0.03366 & 0.00000 & 0.00112 \\
-0.04703 & 0.93332 & 0.00188 & 0.00388 & 0.00000 & 0.00135 \\
0.00919 & -0.11074 & 0.94637 & 3.19274 & 0.00000 & -0.00062 \\
-0.00647 & -0.14191 & -0.03446 & 0.94410 & 0.00000 & -0.00141 \\
0.00081 & 0.00383 & -0.00007 & -0.00094 & 1.00000 & -0.00750 \\
0.00000 & 0.00000 & 0.00000 & 0.00000 & 0.00000 & 1.00000
\end{tabular}

In the calculations of the above matrix elements we assumed that there are no errors in the measurement of the position and angle of rays at the entrance and exit of the magnet. 
In order to calculate the error introduced on the above matrix elements because of the error in the measurement of the position and angle of the rays at the entrance and exit of the magnet, we assume an error, (uniformly distributed between $\pm 0.025 \mathrm{~cm}$ and \pm 0.025 $\mathrm{mrad}$ ) in the measurement of the position and angle of all the rays respectively. A random error is selected from the uniform distribution and is introduced into the position and angle of all the rays. Using the new ray coordinates a new calculation of the first order matrix elements is performed. A total of 50 first order matrices ("error_R_matrices") were calculated. The average value of the matrix elements corresponding to fifty matrices subject to random errors in the ray coordinates is shown in the "Average-R-matrix" below.

\begin{tabular}{rccccc}
\multicolumn{7}{c}{ *TRANSFORM* 1 Units in m,rads } \\
0.90243 & 3.16911 & -0.02164 & 0.02930 & 0.00000 & 0.01036 \\
-0.04675 & 0.93740 & 0.00162 & 0.00555 & 0.00000 & 0.00289 \\
0.00960 & -0.17661 & 0.93332 & 3.32801 & 0.00000 & 0.00742 \\
-0.00723 & -0.14177 & -0.03430 & 0.94886 & 0.00000 & -0.00039 \\
0.00081 & 0.00383 & -0.00007 & -0.00094 & 1.00000 & -0.00750 \\
0.00000 & 0.00000 & 0.00000 & 0.00000 & 0.00000 & 1.00000
\end{tabular}

The Standard deviation from the average value of each matrix element shown in the "Average-R-matrix" above was calculated from the corresponding elements of the 50 "error_R_matrices" and is shown in the "Standard_dev_R_matrix" below.

\begin{tabular}{llllll}
\multicolumn{7}{c}{ *TRANSFORM* 1 Units in m,rads } \\
0.04732 & 0.41756 & 0.04759 & 0.48599 & 0.00000 & 0.18252 \\
0.00465 & 0.05626 & 0.00400 & 0.04408 & 0.00000 & 0.01907 \\
0.03892 & 0.42696 & 0.04591 & 0.43154 & 0.00000 & 0.16644 \\
0.00415 & 0.04755 & 0.00429 & 0.04671 & 0.00000 & 0.01773 \\
0.00002 & 0.00007 & 0.00000 & 0.00002 & 0.00000 & 0.00000 \\
0.00000 & 0.00000 & 0.00000 & 0.00000 & 0.00000 & 0.00000
\end{tabular}

By comparing the matrix elements of the "Ideal R_matrix" with the standard deviation of the corresponding matrix elements shown in the "Standard_dev_R_matrix" we notice that the magnitude of the standard deviation of few of the first order matrix elements like $\mathrm{R}_{14}, \mathrm{R}_{16}$ etc. is larger than the value of the corresponding matrix element appearing in the "Ideal R_matrix".

We have not yet experimentally determined the actual error in the measurement of the position and angle of individual rays. In this section we have assumed that a reasonable maximum error in the measurements of the position and direction of a ray is $\pm 0.025 \mathrm{~cm}$ and $\pm 0.025 \mathrm{mrad}$ respectively. This measurement error in the position and direction of a given ray is uniformly distributed between $\pm 0.025 \mathrm{~cm}$ and $\pm 0.025 \mathrm{mrad}$ and each ray is assigned an error which is randomly selected between these two limits of $\pm 0.025 \mathrm{~cm}$ and $\pm 0.025 \mathrm{mrad}$. This error in the measurement of the position and direction of the rays yielded an error in the Transfer-matrix elements which shown in the "Standard dev R matrix" above.

In order to compare the "accuracy", in the determination of the Transfer-matrix elements, of this proposed method to the method that utilizes magnetic field measurements we have attempted to answer the following question: 
"What is the maximum allowed magnetic field error in the measurement of the value of each of the magnetic field component $\left(\mathrm{B}_{\mathrm{x}}, \mathrm{B}_{\mathrm{y}}, \mathrm{B}_{\mathrm{z}}\right)$ that will produce a "Standard dev R matrix" comparable to that generated with the proposed method (of measuring the Transfer-matrix elements) when an error of $\pm 0.025 \mathrm{~cm}$ and $\pm 0.025 \mathrm{mrad}$ is assumed?"

To answer the above question we perform the following calculations:

a) We assume the helical magnet is described by an "ideal magnetic field map" which is identical to the one used in generate the "Ideal $\mathrm{R}$ matrix" above. Thus under ideal conditions of NO errors in position and angle (proposed method) or NO errors in the magnetic fields (magnetic field method) both methods will generate the same "Ideal R matrix".

b) In order to calculate one of the "error_R_matrices" which correspond to the measurement of the magnetic field at each grid point of the map, we assume a maximum magnetic error (same for each field component $B_{x}, B_{y}, B_{z}$ ). This maximum field error $B_{\text {er }}$, is uniformly distributed between the limits $\pm \Delta B_{\text {er }}$. For each particular grid point of the field map, a set of random errors $\left(\Delta B_{x}, \Delta B_{y}\right.$, $\left.\Delta \mathrm{B}_{\mathrm{z}}\right)$ is selected from the uniform distribution and is applied to the actual fields $\left(\mathrm{B}_{\mathrm{x}}, \mathrm{B}_{\mathrm{y}}, \mathrm{B}_{\mathrm{z}}\right)$ of each grid point.

c) Using the field map with the assigned errors we use the computer code SPRAY and we calculate a particular "error_R_matrix". We repeat tasks (b) and (c) above fifty time to obtain fifty "error_R_matrices"

d) An "Average-R-matrix" and a "Standard_dev_R_matrix" are calculated from the fifty "error_R_matrices".

The average value of the matrix elements corresponding to 50 "error_R_matrices" subject to random errors in the field component $\left(\mathrm{B}_{\mathrm{x}}, \mathrm{B}_{\mathrm{y}}, \mathrm{B}_{\mathrm{z}}\right)$ is shown in the "Average- $\mathrm{R}-$ matrix" below.

\begin{tabular}{rrrrrr}
\multicolumn{7}{c}{ *TRANSFORM * 1 Units in m,rads } \\
0.92354 & 3.12263 & -0.01294 & -0.04553 & 0.00000 & 0.00030 \\
-0.04564 & 0.94582 & -0.00377 & -0.01007 & 0.00000 & 0.00036 \\
0.00985 & -0.09272 & 0.94470 & 3.20434 & 0.00000 & 0.00050 \\
-0.00526 & -0.12742 & -0.03676 & 0.95363 & 0.00000 & -0.00081 \\
0.00078 & 0.00373 & -0.00004 & -0.00081 & 1.00000 & -0.00752 \\
0.00000 & 0.00000 & 0.00000 & 0.00000 & 0.00000 & 1.00000
\end{tabular}

The Standard deviation from the average value of each matrix element shown in the "Average-R-matrix" above was calculated from the corresponding elements of the 50 "error_R_matrices" and is shown in the "Standard_dev_R_matrix" below.

\begin{tabular}{llllll}
\multicolumn{7}{c}{ *TRANSFORM* 1 Units in m,rads } \\
0.03567 & 0.10437 & 0.03236 & 0.06906 & 0.00000 & 0.00226 \\
0.02146 & 0.08460 & 0.02524 & 0.06254 & 0.00000 & 0.00215 \\
0.04048 & 0.11221 & 0.03288 & 0.08305 & 0.00000 & 0.00446 \\
0.02424 & 0.14219 & 0.02088 & 0.09236 & 0.00000 & 0.00328 \\
0.00042 & 0.00235 & 0.00040 & 0.00077 & 0.00000 & 0.00007 \\
0.00000 & 0.00000 & 0.00000 & 0.00000 & 0.00000 & 0.00000
\end{tabular}

The comparison of the "Standard_dev_R_matrices" as calculated with the two methods ("position and direction of rays method" and "field measurement method") shows that 
both methods yields a comparable error in the calculation of the Transfer-matrix elements when a maximum error of $\pm 0.025 \mathrm{~cm}$ and $\pm 0.025 \mathrm{mrad}$ is assumed in the position and direction of the rays (proposed method) and an error of $\pm \Delta \mathrm{B}_{\text {er }}$ is assumed in the measurement of the magnetic fields (magnetic field method).

\section{Summary}

A method to determine experimentally the focusing properties of a magnet has been discussed. This method can determines the first order matrix elements ( $R$ _matrix) of a magnet and the most "significant" higher order matrix elements. The error in the determination of the first order matrix elements is "reasonably small" for the measured R_matrix to be used in various calculations which may involve the focusing properties of the measured magnet.

\section{References}

[1] T. Roser, AIP Conf. Proc. 187 (1988) 1221.

[2] H.Huang, et. al. Phys. Rev. Lett. 73 (1994) 2982

[3] Design Study of a Normal Conducting Helical Snake for AGS Junpei Takano, et. al.

[4] Study of the focusing properties of a warm Helical Snake.

N. Tsoupas et. al. Technical Note \#??????

[5] N Tsoupas et. al. BNL Technical Note No. 26 BNL/NPB-86-84

[6] The computer code "SPRAY" (Spin and Particle Ray-trace) N. Tsoupas BNL private communication.

[7] A $60 \mathrm{kV}$ ion source for magnetic mapping N.Tsoupas Private communication (work to be published).

[8] Vector Field Inc. 